\title{
Fusion-dependent formation of lipid nanoparticles containing macromolecular payloads
}

\author{
Kulkarni, Jayesh A ; Witzigmann, Dominik ; Leung, Jerry ; van der Meel, Roy ; Zaifman, Josh ;
} Darjuan, Maria M ; Grisch-Chan, Hiu Man ; Thöny, Beat ; Tam, Yuen Yi C ; Cullis, Pieter R

\begin{abstract}
The success of Onpattro ${ }^{\mathrm{TM}}$ (patisiran) clearly demonstrates the utility of lipid nanoparticle (LNP) systems for enabling gene therapies. These systems are composed of ionizable cationic lipids, phospholipid, cholesterol, and polyethylene glycol (PEG)-lipids, and are produced through rapid-mixing of an ethanolic-lipid solution with an acidic aqueous solution followed by dialysis into neutralizing buffer. A detailed understanding of the mechanism of LNP formation is crucial to improving LNP design. Here we use cryogenic transmission electron microscopy and fluorescence techniques to further demonstrate that LNP are formed through the fusion of precursor, pH-sensitive liposomes into large electron-dense core structures as the $\mathrm{pH}$ is neutralized. Next, we show that the fusion process is limited by the accumulation of PEG-lipid on the emerging particle. Finally, we show that the fusion-dependent mechanism of formation also applies to LNP containing macromolecular payloads including mRNA, DNA vectors, and gold nanoparticles.
\end{abstract}

DOI: https://doi.org/10.1039/C9NR02004G

Posted at the Zurich Open Repository and Archive, University of Zurich

ZORA URL: https://doi.org/10.5167/uzh-194385

Journal Article

Accepted Version

Originally published at:

Kulkarni, Jayesh A; Witzigmann, Dominik; Leung, Jerry; van der Meel, Roy; Zaifman, Josh; Darjuan, Maria M; Grisch-Chan, Hiu Man; Thöny, Beat; Tam, Yuen Yi C; Cullis, Pieter R (2019). Fusiondependent formation of lipid nanoparticles containing macromolecular payloads. Nanoscale, 11(18):90239031.

DOI: https://doi.org/10.1039/C9NR02004G 


\title{
Fusion-Dependent Formation of Lipid Nanoparticles Containing Macromolecular Payloads
}

\author{
Jayesh A. Kulkarni ${ }^{1,2 \S}$, Dominik Witzigmann ${ }^{1 \S}$, Jerry Leung ${ }^{1}$, Roy van der Meel ${ }^{1,3,4}$, Josh \\ Zaifman $^{1,5}$, Maria M. Darjuan ${ }^{1,5}$, Hiu Man Grisch-Chan ${ }^{6}$, Beat Thöny ${ }^{6}$, Yuen Yi C. Tam ${ }^{1,5}$, and \\ Pieter R. Cullis ${ }^{1^{*}}$ \\ ${ }^{\S}$ Contributed equally \\ ${ }^{1}$ Department of Biochemistry and Molecular Biology, University of British Columbia, 2350 \\ Health Sciences Mall, Vancouver, British Columbia, Canada, V6T 1 Z3 \\ ${ }^{2}$ Department of Medical Genetics, University of British Columbia, 950 West $28^{\text {th }}$ Avenue, \\ Vancouver, British Columbia, V5Z 4H4 \\ ${ }^{3}$ Department of Clinical Chemistry and Haematology, University Medical Center Utrecht, \\ Utrecht, The Netherlands \\ ${ }^{4}$ Laboratory of Chemical Biology, Department of Biomedical Engineering and Institute for \\ Complex Molecular Systems, Eindhoven University of Technology, Eindhoven, The Netherlands \\ ${ }^{5}$ Integrated Nanotherapeutics, 6190 Agronomy Road, Vancouver, British Columbia, Canada, \\ V6T 123
}

${ }^{6}$ Division of Metabolism, University Children's Hospital Zurich and Children's Research Centre, Steinwiesstrasse 75, 8032 Zurich, Switzerland

\section{Prepared as per Nanoscale}

*Correspondence to: Pieter R Cullis, Tel: +1 (604)-822-4144, Fax: +1 (604)-822-4843, Email: pieterc@mail.ubc.ca 
Present Address: Life Sciences Institute, University of British Columbia, 2350 Health Sciences Mall, Vancouver, British Columbia, Canada, V6T 1Z3

\begin{abstract}
The success of Onpattro ${ }^{\mathrm{TM}}$ (patisiran) clearly demonstrates the utility of lipid nanoparticle (LNP) systems for enabling gene therapies. These systems are composed of ionizable cationic lipids, phospholipid, cholesterol, and polyethylene glycol (PEG)-lipids, and are produced through rapidmixing of an ethanolic-lipid solution with an acidic aqueous solution followed by dialysis into neutralizing buffer. A detailed understanding of the mechanism of LNP formation is crucial to improving LNP design. Here we use cryogenic transmission electron microscopy and fluorescence techniques to further demonstrate that LNP are formed through the fusion of precursor, $\mathrm{pH}-$ sensitive liposomes into large electron-dense core structures as the $\mathrm{pH}$ is neutralized. Next, we show that the fusion process is limited by the accumulation of PEG-lipid on the emerging particle. Finally, we show that the fusion-dependent mechanism of formation also applies to LNP containing macromolecular payloads including mRNA, DNA vectors, and gold nanoparticles.
\end{abstract}

\title{
KEYWORDS
}

lipid nanoparticles, gene therapy, lipid biophysics, cryo-TEM, nanomedicine, mRNA, mechanism 


\section{INTRODUCTION}

Onpattro $^{\mathrm{TM}}$ (patisiran) is the first RNA interference therapeutic approved by the FDA and EMA. ${ }^{1,2}$ The technology enabling the delivery of therapeutic short interfering RNA (siRNA) is based on lipid nanoparticles (LNP). These systems are composed of ionizable cationic lipids (e.g. 2,2-dilinoleyl-4-(2-dimethylaminoethyl)-[1,3]-dioxolane $\quad(\mathrm{KC} 2)^{3} \quad$ or heptatriaconta-6,9,28,31tetraen-19-yl-4-(dimethylamino)butanoate $\left.(\mathrm{MC} 3)^{4}\right)$, phospholipid, cholesterol and polyethylene glycol-lipid. ${ }^{5,6}$ LNP are generated through rapid-mixing techniques ${ }^{7}$ where an ethanolic lipid solution is diluted into an acidic aqueous phase containing the anionic macromolecules. We have previously shown that LNP prepared in this way display high entrapment efficiencies $(>85 \%)$ for anionic macromolecules such as siRNA $^{8}$ and plasmid DNA (pDNA) ${ }^{9}, 10$, or colloidal gold nanoparticles (GNP). ${ }^{10}$

LNP-siRNA systems display an electron-dense core as observed by cryogenic transmission electron microscopy (cryo-TEM). ${ }^{11,12}$ It was originally suggested that this electron-dense core reflects inverted micellar structures generated by the association of ionizable cationic lipid with the anionic payload. ${ }^{12}$ These hydrophobic structures were hypothesized to aggregate and then become coated with a monolayer of polar lipids such as PEG-lipids as these lipids reach their solubility limits in the ethanol/water mixture. This hypothesis implied that the final LNP structure is established during (and as a result of) the rapid-mixing process. . $^{2}$, 8, 12,13 Recently, we reexamined the LNP-siRNA structure, demonstrating that the LNP electron-dense core morphology does not consist of inverted micelles but reflects an oil core consisting primarily of neutral ionizable lipid, ${ }^{11}$ and suggested that LNP-siRNA formulations are generated through fusion of smaller particles that occurs after the rapid-mixing procedure. While much of that work was 
performed with the ionizable lipid $\mathrm{KC} 2$, the same structures and mechanism of formation are seen for LNP with the Onpattro ${ }^{\mathrm{TM}}$ composition (containing MC3; Supporting Figure S1).

In this work, we further elucidate the mechanism of LNP formation following the rapidmixing step for LNP systems using an electron microscopy- and fluorescence-based approach. We examine the role of PEG-lipids in limiting the fusion process and extend our investigation of LNP morphology to include larger payloads such as messenger RNA (mRNA), minicircle DNA (mcDNA), pDNA, or GNP. Results obtained support the proposal that LNP formation occurs through a pH-dependent fusion process that occurs subsequent to the rapid mixing event, and that the formation of LNP with large payloads builds on formation of LNP formed at $\mathrm{pH} 4$ that contain mRNA, mcDNA or pDNA. 


\section{MATERIALS AND METHODS}

\section{Materials}

The lipids 1,2-distearoyl-sn-glycero-3-phosphorylcholine (DSPC) and 1,2-distearoyl-sn-glycero3-phosphoethanolamine-N-[methoxy(polyethylene glycol)-2000] (ammonium salt) (PEG-DSPE) were purchased from Avanti Polar Lipids (Alabaster, AL). The ionizable amino-lipid 2,2dilinoleyl-4-(2-dimethylaminoethyl)-[1,3]-dioxolane (KC2) was synthesized by Biofine International (Vancouver, BC). Cholesterol was purchased from Sigma-Aldrich (St. Louis, MO). Heptatriaconta-6,9,28,31-tetraen-19-yl 4-(dimethylamino)butanoate (MC3) ${ }^{4}$ and (R)-2,3bis(tetradecyloxy)propyl-1-(methoxy polyethylene glycol 2000) carbamate (PEG-DMG) ${ }^{14}$ were synthesized as previously described. Lipophilic indocarbocyanine dyes 3,3'dioctadecyloxacarbocyanine perchlorate (DiO) and 1,1'-dioctadecyl-3,3,3',3'tetramethylindocarbocyanine perchlorate (DiI) were purchased from ThermoFisher Scientific (Waltham, MA). TEM grids and gold nanoparticles were purchased from Ted Pella, Inc. (Redding, CA). mRNA encoding firefly luciferase was purchased from TriLink Biotechnologies (San Diego, CA). pDNA encoding TdTomato ${ }^{15}$ was purchased from Addgene (Cambridge, MA) and prepared using a Qiagen Endotoxin-free Giga prep kit (Hilden, Germany). Minicircle DNA (mcDNA) was generated by transforming the mcDNA producer plasmids into E. coli ZYCY10P3S2T and purification of resulting mcDNA vectors using the Qiagen Endotoxin-free plasmid purification kit as previously described. ${ }^{16}$ siRNA against firefly luciferase ${ }^{17}$ was purchased from IDT (Coralville, IA). 
LNP were prepared as previously described." ${ }^{91}$ Briefly, lipid components (KC2, Chol, DSPC, and PEG-lipid) at appropriate ratios were dissolved in ethanol to a concentration of 10-15 mM total lipid. For fluorescence resonance energy transfer (FRET) experiments: non-exchangeable donor or acceptor lipid tracers (DiO or DiI, respectively) were added to lipid mixtures at a concentration of $0.2 \mathrm{~mol} \%$.

The aqueous phase consisted of $25 \mathrm{mM}$ sodium acetate $\mathrm{pH} 4$ buffer. The two solutions were mixed through a T-junction mixer ${ }^{18,19}$ at a total flow rate of $20 \mathrm{~mL} / \mathrm{min}$, and a flow rate ratio of 3:1 v/v (corresponding to $15: 5 \mathrm{~mL} / \mathrm{min}$ aqueous:organic phase). Unless otherwise specified, the resulting suspension was subsequently dialysed against 1000-fold volume of the same sodium acetate $\mathrm{pH} 4$ buffer or against phosphate buffered saline (PBS pH 7.4).

For post-formation PEG-insertion studies: LNPs were prepared with KC2, DSPC and Chol (no PEG-lipid) using the rapid-mixing technique described above, where the ethanolic lipid phase was combined with a $25 \mathrm{mM}$ sodium acetate $\mathrm{pH} 4$ buffer. Following dialysis into the same $\mathrm{pH} 4$ buffer to remove ethanol the resulting LNPs were concentrated to achieve a final concentration of $2.5 \mathrm{mM}$ total lipid. PEG-lipid dissolved in ethanol was added to appropriate amounts to achieve 0.5-2.5 mol\% PEG-lipid. At all molar fractions of PEG, the total amount of ethanol in the mixture was $1 \% \mathrm{v} / \mathrm{v}$. The solutions were pipette-mixed and the resulting mixture was dialysed against 1000-fold volume of PBS overnight. 
FRET-based fusion assay

LNP-DiO, LNP-DiI or LNP-DiO/LNP-DiI mixtures (equimolar concentrations) at $\mathrm{pH} 4$ or $\mathrm{pH} 7.4$ were diluted using sodium acetate $\mathrm{pH} 4$ buffer or PBS pH 7.4. Samples were excited at $470 \mathrm{~nm}$ and the emission was collected between $505-650 \mathrm{~nm}$. FRET data from each set of experiment were normalized to emission of donor probe (LNP-DiO) under identical conditions. Spectroscopic evidence for FRET could be observed by decrease of fluorescence at $505 \mathrm{~nm}$ and increase at $570 \mathrm{~nm}$.

Preparation of LNP containing nucleic acid

LNP-nucleic acid were prepared as previously described. ${ }^{9,11}$ Briefly, lipid components (KC2, Chol, DSPC, and PEG-lipid) at appropriate ratios were dissolved in ethanol to a concentration of 10-15 mM total lipid. Purified nucleic acid polymers were dissolved in $25 \mathrm{mM}$ sodium acetate $\mathrm{pH} 4$ buffer to achieve a ratio of $0.029 \mathrm{mg}$ nucleic acid per $\mu \mathrm{mol}$ lipid (corresponding to amineto-phosphate $(\mathrm{N} / \mathrm{P})$ ratio of 6$)$ unless otherwise specified. The two solutions were mixed through a T-junction mixer ${ }^{18,19}$ at a total flow rate of $20 \mathrm{~mL} / \mathrm{min}$, and a flow rate ratio of $3: 1 \mathrm{v} / \mathrm{v}$ (corresponding to $15: 5 \mathrm{~mL} / \mathrm{min}$ aqueous:organic phase). The resulting suspension was subsequently dialysed against the same sodium acetate $\mathrm{pH} 4$ buffer or directly against PBS pH 7.4.

\section{Preparation of LNP containing gold nanoparticles (GNP)}

LNP-GNP were prepared using a modified process from that described elsewhere. ${ }^{10}$ Briefly, lipid components (KC2, Chol, DSPC, and PEG-lipid) at appropriate ratios were dissolved in ethanol to a concentration of 10-15 mM total lipid. $5 \mathrm{~nm}$ GNP with a tannic-acid surface modification (Ted Pella, Redding, CA) were suspended in $25 \mathrm{mM}$ sodium acetate $\mathrm{pH} 4$ to a concentration of 
$2.2 \times 10^{13} \mathrm{GNP} / \mu \mathrm{mol}$ lipid. In order to maintain the same charge ratio (negative charges of the GNP to positively charged lipid), $12 \mathrm{~nm}$ GNP were formulated at $3.82 \times 10^{12} \mathrm{GNP} / \mu \mathrm{mol}$ lipid. This number was generated based on maintaining a constant ratio of surface area of GNP to amount of lipid (1.73 x $10^{15} \mathrm{~nm}^{2} / \mu \mathrm{mol}$ lipid). The two solutions were mixed through a T-junction mixer 18,19 at a total flow rate of $20 \mathrm{~mL} / \mathrm{min}$, and a flow rate ratio of $3: 1 \mathrm{v} / \mathrm{v}$ (corresponding to $15: 5$ $\mathrm{mL} / \mathrm{min}$ aqueous:organic phase). The resulting suspension was subsequently dialysed against the same sodium acetate $\mathrm{pH} 4$ buffer or directly against PBS $\mathrm{pH}$ 7.4. For studies including a mixture of 5 and $12 \mathrm{~nm} \mathrm{GNP,} \mathrm{the} \mathrm{LNP} \mathrm{systems} \mathrm{were} \mathrm{always} \mathrm{mixed} \mathrm{after} \mathrm{the} \mathrm{rapid-mixing} \mathrm{process.}$

\section{Cryogenic transmission electron microscopy (cryo-TEM)}

Cryo-TEM was performed as previously described. ${ }^{11}$ LNP suspensions were concentrated to a final concentration of $20-25 \mathrm{mg} / \mathrm{mL}$ of total lipid and added to glow-discharged copper grids (3-5 $\mu \mathrm{L}$ ), and plunge-frozen using a FEI Mark IV Vitrobot (FEI, Hillsboro, OR) to generate vitreous ice. Grids were stored in liquid nitrogen until imaged. Grids were moved into a Gatan $70^{\circ}$ cryotilt transfer system pre-equilibrated to at least $-180^{\circ} \mathrm{C}$ prior to insertion into the microscope. An FEI LaB6 G2 TEM (FEI, Hillsboro, OR) operating at $200 \mathrm{kV}$ under low-dose conditions was used to image all samples. A bottom-mount FEI Eagle $4 \mathrm{~K}$ CCD camera was used to capture all images. All samples (unless otherwise stated) were imaged at a 55,000x magnification with a nominal under-focus of 1-2 $\mu \mathrm{m}$ to enhance contrast. Sample preparation and imaging was performed at the UBC Bioimaging Facility (Vancouver, BC). 


\section{Analysis of LNPS}

Cryo-TEM micrographs obtained for each sample were characterized for particle size (as compared by length to the scale bar), performed by manual counting of at least 150 LNPs to account for scattering interference from different morphology. Such an approach has been shown to closely correlate with the number-weighted average produced by dynamic light scattering. ${ }^{20}$ Similarly, the fraction of loaded LNPs was performed manually. Lipid concentrations were measured using the Cholesterol E Total-Cholesterol assay (Wako Diagnostics, Richmond, VA). RNA entrapment was measured using the procedure described elsewhere. ${ }^{21}$

\section{Statistical analysis}

Statistical analyses were performed for all quantitative data using GraphPad. Where applicable, two-way ANOVAs were performed using the Tukey multiple comparison test and confidence level of 0.001. Similarly, where applicable an unpaired t-test was performed. All significance values are provided in the figure legends or in the Supporting Information. 


\section{RESULTS and DISCUSSION}

FRET and Cryo-TEM studies demonstrate fusion of precursor liposomes

Initial studies were focused on characterizing the fusion events following the rapid mixing of $\mathrm{KC} 2 / \mathrm{DSPC} / \mathrm{Chol} / \mathrm{PEG}-\mathrm{lipid}(50 / 10 / 39 / 1 \mathrm{~mol} \%)$ in ethanol with an aqueous stream of $25 \mathrm{mM}$ acetate buffer $\mathrm{pH} 4$ buffer to form "empty" LNP systems. The resulting mixture was then dialyzed against the acetate buffer to remove ethanol, or against phosphate buffered saline (PBS) $\mathrm{pH} 7.4$ to remove solvent and neutralize the $\mathrm{pH}$. In agreement with previous studies, ${ }^{11}$ the empty LNP display small bilayer structures at $\mathrm{pH} 4$ where $\mathrm{KC} 2(\mathrm{pKa} \sim 6.7)$ is protonated but form larger electron-dense structures at $\mathrm{pH} 7.4$ (Figure 1A/B). In order to directly demonstrate that the larger structures result from fusion of structures formed at $\mathrm{pH} 4$, LNP were formulated with nonexchangeable fluorescence resonance energy transfer (FRET) lipid tracers; either a donor probe (DiO; green; Ex 484 nm, Em $501 \mathrm{~nm}$ ) or an acceptor probe (DiI; red; Ex 549 nm, Em 565 nm), which are able to act as a FRET pair when in close proximity, ${ }^{22}$ i.e. present in the same LNP. If the particles fuse, a FRET signal would be expected as emission at $565 \mathrm{~nm}$, when excited at 470 nm. As shown in Figure 1C (top left), when the two formulations are combined at $\mathrm{pH} 4$, the emission profile was an additive effect of two separate LNP formulations (no FRET). However, when LNP-DiO and LNP-DiI were combined at $\mathrm{pH} 4$ and neutralized with addition of PBS, a clear FRET signal was observed (Figure 1C, top right). In order to determine if lipid mixing occurred after the $\mathrm{pH}$ was raised to 7.4, LNP-DiO and LNP-DiI at $\mathrm{pH} 7.4$ were combined and then the $\mathrm{pH}$ was lowered to 4 (Figure 1C, bottom left) - no FRET signal was observed. Similarly, LNP combined at pH 7.4 and diluted with the same buffer did not display a FRET signal (Figure 1C, bottom right). These data provide clear evidence that raising the $\mathrm{pH}$ from 4 to 7.4 promotes fusion of particles and that no lipid exchange occurs following this fusion process. 
$\mathbf{A}$

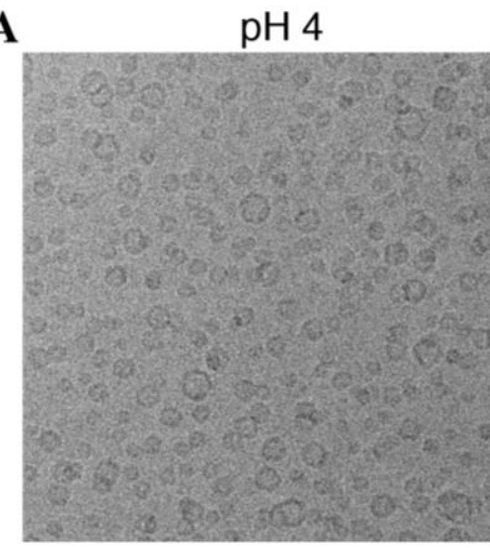

C

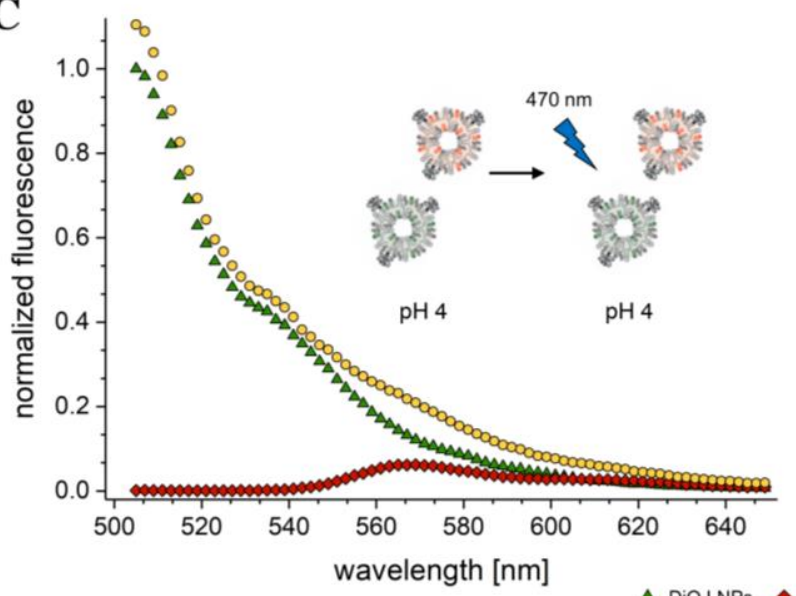

$\Delta$ DiOLnPs

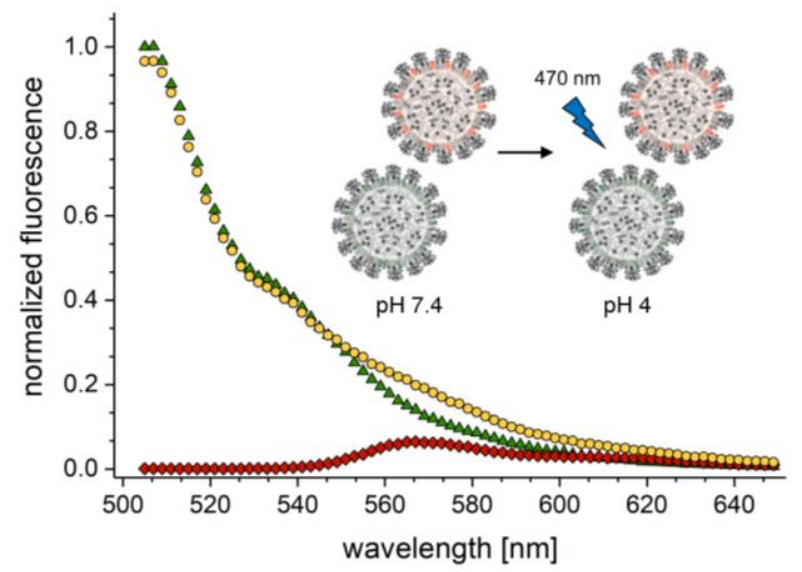

$\mathrm{pH} 7.4$

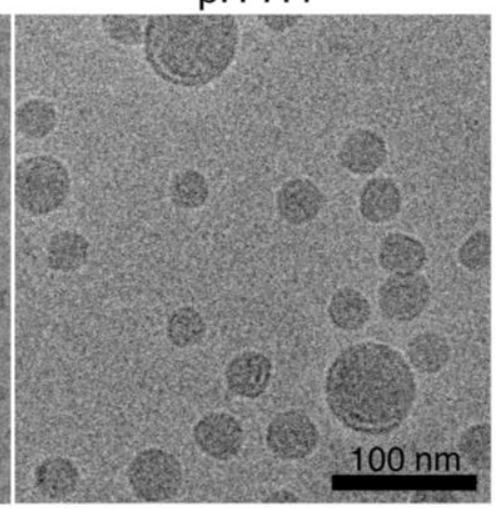

B

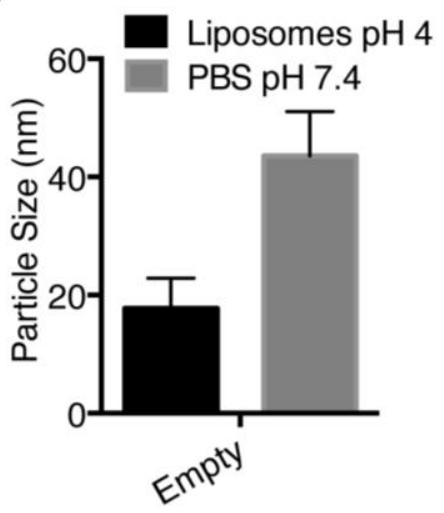

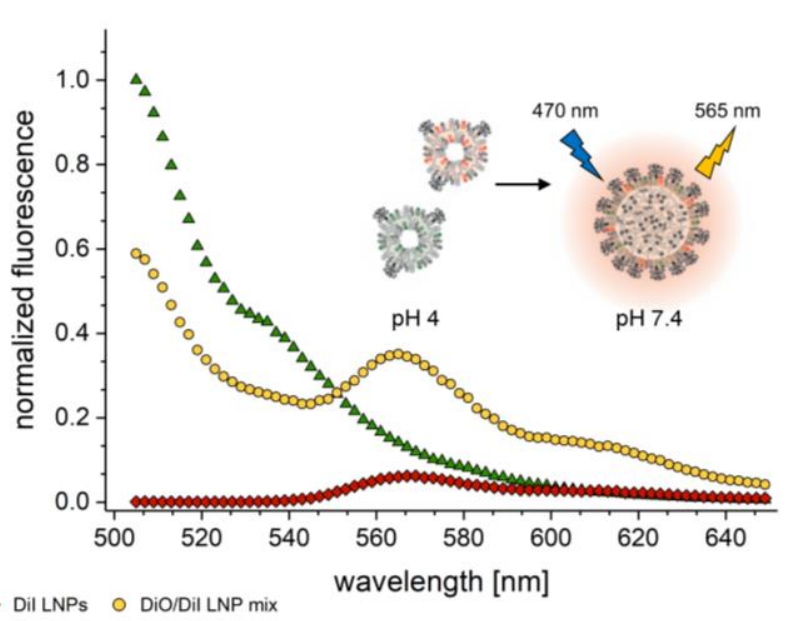

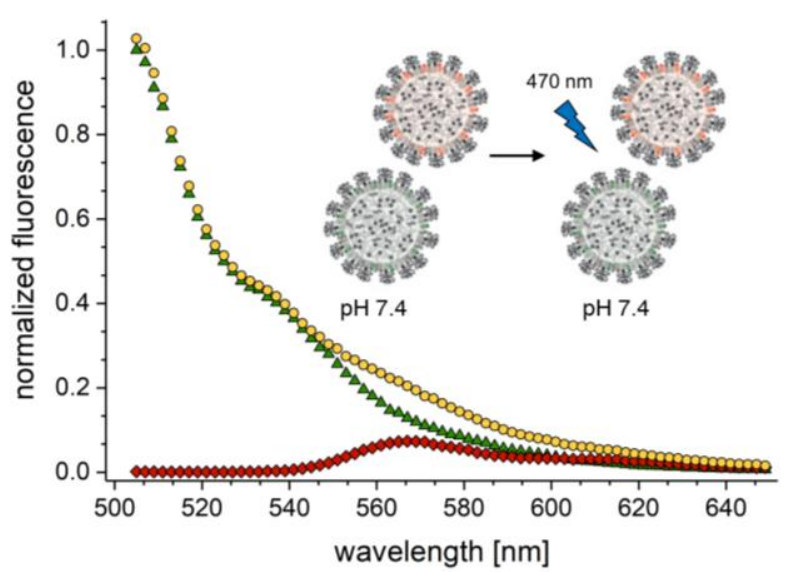

Figure 1. Empty LNP observed as electron-dense structures are generated through fusion of smaller vesicular structures. (A) Empty LNP composed of $\mathrm{KC} 2 / \mathrm{Chol} / \mathrm{DSPC} / \mathrm{PEG}-\mathrm{lipid}$ $(50 / 10 / 39 / 1 \mathrm{~mol} \%)$ were generated at $\mathrm{pH} 4$ and dialyzed into $\mathrm{pH} 4$ buffer to remove solvent, or dialysed into PBS pH 7.4 to remove solvent and neutralize the $\mathrm{pH}$. Scale bar $=100 \mathrm{~nm}$. (B) Particle sizes of LNP at $\mathrm{pH} 4$ and $\mathrm{pH} 7.4$ as determined by manual measurement of 200 particles. Unpaired t-test, $\mathrm{p}<0.0001$. (C) LNP-DiO (donor) and LNP-DiI (acceptor) systems were combined under different conditions to determine if particle fusion generated a FRET signal. Top left: Formulations prepared in $\mathrm{pH} 4$ were combined in $\mathrm{pH} 4$ buffer. Top right: Formulations prepared in $\mathrm{pH} 4$ buffer were combined and the $\mathrm{pH}$ was neutralized ( $\mathrm{pH}$ 7.4). Note this is the only condition which resulted 
in FRET. Bottom left: Formulations prepared in PBS were combined in $\mathrm{pH} 4$ buffer. Bottom right: Formulations prepared in PBS were combined and diluted in PBS. Each panel has an inset describing the experiment.

\section{PEG-lipids limit fusion and dictate particle size}

We next examined the role of PEG-lipids in determining the equilibrium size of these LNP. Previous work has shown that PEG-lipids reside primarily on the LNP surface where they exhibit a large area per molecule at the polar-non-polar interface of approximately $26 \mathrm{~nm}^{2}$ (for $\left.\mathrm{PEG}_{2000}\right)^{8}$. As a result, the proportion of PEG-lipid dictates the size of the LNP as the concentration of PEGlipid on the surface approaches a critical level that inhibits further fusion. ${ }^{8,} 12,21$ Here, we show that empty LNP composed of KC2/DSPC/Chol/PEG-lipid (50/10/37.5-39.5/0.5-2.5 mol\%) form bilayer structures at pH 4 (Figure 2A). Regardless of PEG-lipid content, the particle size at pH 4 is $\sim 17 \mathrm{~nm}$ (Figure $2 \mathrm{~B})$, but ranges from $31.9 \mathrm{~nm}(2.5 \mathrm{~mol} \%)$ to $58.1 \mathrm{~nm}(0.5 \mathrm{~mol} \%)$ at $\mathrm{pH} 7.4$ (Figure 2C), indicating that the PEG-lipid exerts its size-limiting role during neutralization of the $\mathrm{pH}$. In order to demonstrate this more directly, LNPs composed of only KC2, DSPC and cholesterol (without PEG) were formulated by ethanol dilution/rapid mixing at $\mathrm{pH} 4$ and dialyzed into the pH 4 buffer to remove solvent. PEG-lipid was then added into the LNP suspension from ethanol stocks, briefly mixed and the $\mathrm{pH}$ raised to $\mathrm{pH} 7.4$ through dialysis. The resulting particle sizes were identical to particles where PEG-lipid is included in the initial ethanolic lipid phase (Fig 2C). 


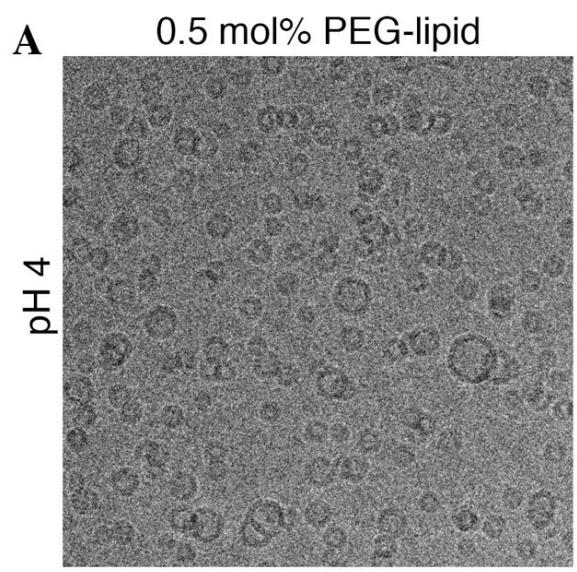

1.5 mol\% PEG-lipid

$2.5 \mathrm{~mol} \%$ PEG-lipid
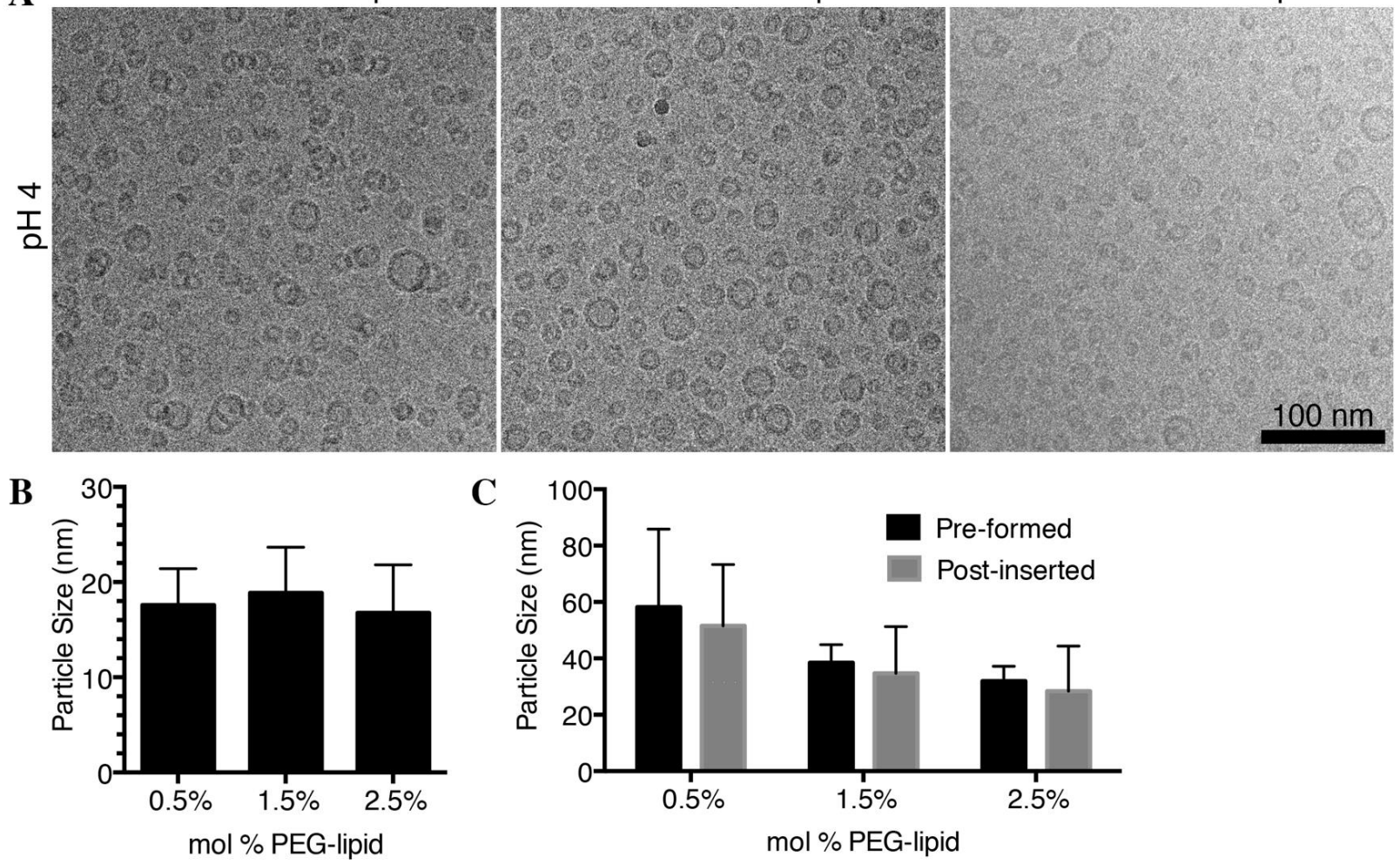

Figure 2. PEG-lipid limits the number of particles that fuse to form the final LNP suspension at pH 7.4. (A) LNP composed of $\mathrm{KC} 2 / \mathrm{DSPC} / \mathrm{Chol} / \mathrm{PEG}-\mathrm{lipid}$ at molar ratios of 50/10/37.539.5/0.5-2.5 (respectively) were prepared in $\mathrm{pH} 4$ buffer through rapid-mixing, and dialyzed into $\mathrm{pH} 4$ buffer to remove solvent. Scale bar $=100 \mathrm{~nm}$. (B) Particle sizes of LNP at pH 4 as determined by manual measurement of 200 particles. (C) Particle size of LNP prepared at $\mathrm{pH} 7.4$ by addition of PEG-lipid at various stages of LNP formation. Formulations composed of KC2, DSPC, and Chol (with or without PEG-lipid) were generated using the rapid-mixing method. The pre-formed LNP mixture contained the appropriate amount of PEG-lipid in the ethanolic phase during rapidmixing (black bars). For another set of formulations (termed post-inserted), particles were prepared without PEG-lipid and dialysed into $\mathrm{pH} 4$ buffer to remove solvent. PEG-lipid was then added into the LNP suspension from ethanol stocks (total ethanol content was $1 \% \mathrm{v} / \mathrm{v}$ ) and dialysed against PBS overnight (grey bars). The resulting particles were analysed by DLS. Results indicate number mean \pm standard deviation. 
Since the area per membrane lipid at the membrane-water interface is dependent on the size of the headgroup, ${ }^{23}$ it would be expected that the area per molecule of the PEG-lipids will depend on the size of the PEG-moiety. LNPs were formulated with PEG-lipid covering the PEG molecular weight range of $350-3000 \mathrm{~g} / \mathrm{mol}$ (at molar fractions of $0.25-5 \mathrm{~mol} \%$ ). As shown in Supporting Figure 2, decreasing the size of the PEG-chain results in significantly larger particles, and increasing the molar fraction of PEG-lipid results in smaller particles. Using the mathematical model described previously, ${ }^{8}$ the corresponding area per PEG-lipid was determined to be 1.3-2 $\mathrm{nm}^{2}$ for $\mathrm{PEG}_{350}$ (large size distributions), $6.5 \mathrm{~nm}^{2}$ for $\mathrm{PEG}_{750}, 26 \mathrm{~nm}^{2}$ for $\mathrm{PEG}_{2000}$ (as previously observed) $)^{8}$, and $45 \mathrm{~nm}^{2}$ for $\mathrm{PEG}_{3000}$.

\section{LNP formulations of $m R N A, m c D N A$ and $p D N A$ are formed through fusion}

It has been demonstrated previously that the ethanol-dilution rapid-mixing formulation process can also be applied to efficiently encapsulate large RNA and DNA payloads such as mRNA $^{10,24}$ and plasmid DNA. ${ }^{9,} 10$ It may be expected that the large size of these molecules will influence the size of the LNP formed at $\mathrm{pH} 4$ that contain nucleic acid. LNP were formulated with mRNA (1.9 kb), mcDNA (1.4 and $3.4 \mathrm{~kb}$-pairs) and plasmid DNA (5.6 kb-pairs) and characterized by cryo-TEM. It was found that LNP at $\mathrm{pH} 4$ displayed two distinct populations: small liposomal structures and larger electron-dense particles (Figure 3A). The electron-dense particles at $\mathrm{pH} 4$ were marginally smaller than the electron-dense particles observed at $\mathrm{pH} 7.4$ (Figure 3B). The obvious difference between siRNA and pDNA is the size of the 21 bp duplex (42 negative charges) as compared to $5.6 \mathrm{kbp}(11,200$ negative charges). At an amine-to-phosphate (N/P) ratio of 1.5 (shown to be necessary to fully condense $\mathrm{pDNA}^{25}$ ), this corresponds to $1.68 \times 10^{4}$ ionizable cationic lipids per pDNA. Assuming a lipid density of $0.9 \mathrm{~g} / \mathrm{mL}$, this corresponds to a lipid volume 
of $3.66 \times 10^{4} \mathrm{~nm}^{3}$ and a plasmid volume of $4.36 \times 10^{3} \mathrm{~nm}^{3} /$ plasmid. The resultant LNP would be $42.8 \mathrm{~nm}$ in diameter if it contained one plasmid, and $52.7 \mathrm{~nm}$ if it contained two plasmids. The large electron-dense core structures (at $\mathrm{pH} 4$ ) are $47.3 \mathrm{~nm}$ in diameter and likely contain one or two plasmids per particle. At $\mathrm{pH} 7.4$, the average particle size increased to $59.4 \mathrm{~nm}$. It is probable that the formulation is composed of a mixture of empty and pDNA-loaded LNP, however the cryoTEM approach is unable to distinguish between these particles. It should be noted that for mRNA and mcDNA, the electron-dense structures (at $\mathrm{pH} 4$ ) are larger than expected (calculated diameters for mRNA: $26.6 \mathrm{~nm}$, mcDNA: $29.0 \mathrm{~nm}$ for $1.9 \mathrm{kbp}$ and $36.7 \mathrm{~nm}$ for $3.4 \mathrm{kbp}$ ), this can be attributed to the presence of particles containing multiple mRNAs ${ }^{26}$ or more than one mcDNA vector (i.e. multiple monomers or concatemers). ${ }^{27}$ 


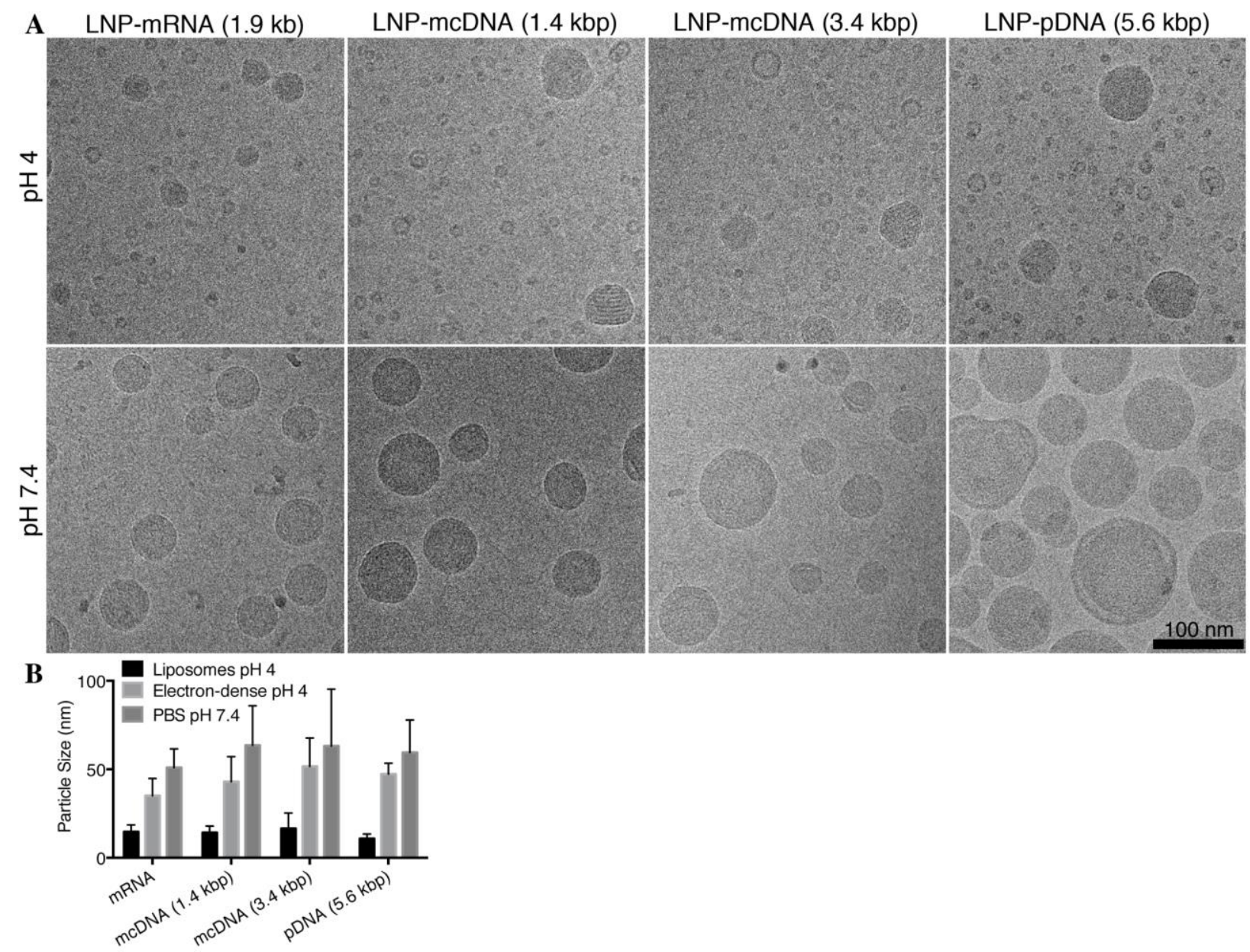

Figure 3. LNP formations of various large nucleic acid polymers undergo fusion as the pH is neutralized to generate the final LNP system. (A) LNP composed of KC2/DSPC/Chol/PEGlipid (50/10/39/1 mol\%) as described elsewhere, ${ }^{9}{ }^{10}$ were prepared with nucleic acids (mRNA, mcDNA (1.4 and $3.4 \mathrm{kbp}$ ), and pDNA (5.6 kpb)) at pH 4 and dialyzed into $\mathrm{pH} 4$ buffer to remove ethanol, or $\mathrm{pH} 7.4$ buffer to remove ethanol and neutralize the $\mathrm{pH}$. The resulting suspensions were concentrated and analyzed by cryo-TEM. Scale bar $=100 \mathrm{~nm}$. (B) Particle sizing data of LNP systems at pH 4 divided into two categories: liposomes and electron-dense core structures, and particles at $\mathrm{pH}$ 7.4. In all cases $\mathrm{n}=200$, except for electron-dense core structures at $\mathrm{pH} 4, \mathrm{n}=$ at least 40. 2-way ANOVA with Tukey's multiple comparisons correction was used to determine statistical difference. All LNP at $\mathrm{pH} 4$ are identical in size $\mathrm{p}>0.5$. For detailed comparisons see Supporting Information. 


\section{GNPs shed light on the role and location of payload in LNP formation}

The final set of studies concerned LNP systems containing GNP where the difference in density allows the negatively charged payload to be readily tracked using cryo-TEM. Here we first examined these structures at $\mathrm{pH} 4$ and $\mathrm{pH} 7.4$ by utilizing $5 \mathrm{~nm}$ GNP (Figure 4A/B, respectively) and $12 \mathrm{~nm}$ GNP (Figure 4C/D, respectively). The negative charge on GNPs results from the tannicacid capping agent. Assuming a constant number of particles per volume, increasing the particle size would result in a different positive-to-negative charge ratio. To account for this, LNP-GNP formulations were generated at a GNP surface area-to-lipid ratio of $1.73 \times 10^{15} \mathrm{~nm}^{2} / \mu \mathrm{mol}$ lipid. LNP systems clearly display evidence of bilayer structures at $\mathrm{pH} 4$ (Figure 4A/C), and much larger electron-dense particles at neutral $\mathrm{pH}$ (Figure 4B/D). This indicates that the particles generated at $\mathrm{pH} 4$ fully entrap the anionic payload during formation and the payload is located in structures that appear as electron-dense. Electron-dense particles at $\mathrm{pH} 4$ are likely those that contain large nucleic acids (i.e. mRNA or DNA vectors), while other particles (i.e. small unilamellar vesicles) are payload-free.

It is of interest to determine whether LNP-GNP particles at $\mathrm{pH} 4$ participated in the fusion process as the $\mathrm{pH}$ is raised or were "fully formed". LNP-GNP formulations were mixed at various stages of particle formation and imaged by cryo-TEM. First, LNP formulations of KC2/DSPC/Chol/PEG-lipid (50/10/39/1 mol\%) containing both 5 and $12 \mathrm{~nm}$ GNP at pH 4 were combined and imaged (Figure 4E). The resulting mixture displayed electron-dense particles containing either 5 or $12 \mathrm{~nm}$ GNPs, but no particles containing both GNP types. Next, LNP-GNP systems at $\mathrm{pH} 7.4$ were combined (Figure 4F). This mixture displayed electron-dense LNP containing either 5 or $12 \mathrm{~nm}$ GNPs suggesting no payload exchange between fully formed LNP. Third, when LNP-GNP formulations containing $5 \mathrm{~nm}$ GNP and $12 \mathrm{~nm}$ GNP were combined 
immediately after rapid-mixing at $\mathrm{pH} 4$ and then dialyzed into PBS $\mathrm{pH} 7.4$ to neutralize and remove solvent, LNP contained either $5 \mathrm{~nm}$ GNP, $12 \mathrm{~nm}$ GNP or a mixture of the two GNP sizes (Figure 4G) indicating that the fusion process is not affected by the presence of encapsulated GNP. A similar experiment was performed combining LNP-GNP systems at $\mathrm{pH} 4$ in the absence of solvent followed by neutralization using dialysis against PBS pH 7.4 (Figure 4H). As in Figure 4G, LNP contained either $5 \mathrm{~nm}$ GNP, $12 \mathrm{~nm}$ GNP or a mixture of both 5 and $12 \mathrm{~nm}$ GNPs. As observed for all other LNP formulations, particles at $\mathrm{pH} 4$ were significantly smaller than those at pH 7.4 (Figure 4I). Importantly, the use of GNPs show clearly that loaded LNP formulations can contain empty LNP, and that the fraction of empty LNP is dictated by the size of the payload (Figure 4J). Thus, formulations entrapping larger nucleic acid sequences are more likely to contain empty LNP in the final formulation at $\mathrm{pH}$ 7.4. 


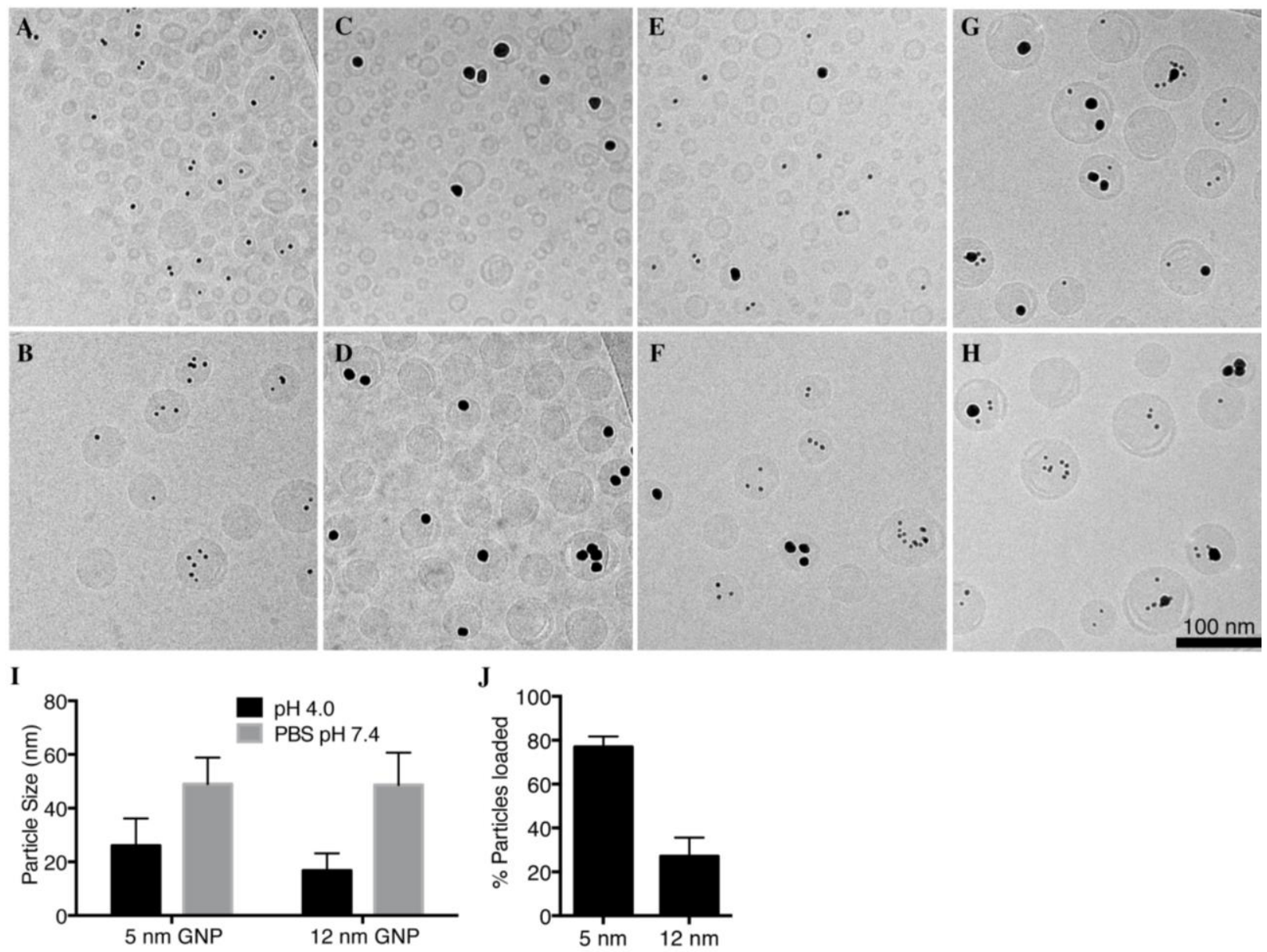

Figure 4. LNP-GNP formulations containing either 5 or $12 \mathrm{~nm}$ GNP suggest particles with GNP at pH 4 participate in further fusion. LNP composed of KC2/DSPC/Chol/PEG-lipid $(50 / 10 / 39 / 1 \mathrm{~mol} \%)$ were prepared with $5 \mathrm{~nm}$ GNP $\left(2.2 \times 10^{13} \mathrm{GNP} / \mathrm{umol}\right.$ lipid $)$ or $12 \mathrm{~nm}$ GNP (3.8 x $10^{12} \mathrm{GNP} / \mathrm{umol}$ lipid) to maintain a constant GNP surface area of $1.73 \times 10^{15} \mathrm{~nm}^{2} / \mathrm{umol}$ lipid. LNP-GNP $_{5 \mathrm{~nm}}$ were imaged by Cryo-TEM at pH 4 (A), and in PBS pH 7.4 (B). LNP-GNP $12 \mathrm{~nm}$ were imaged by Cryo-TEM at $\mathrm{pH} 4$ (C), and at $\mathrm{pH} 7.4$ (D). (E) The two formulations at $\mathrm{pH} 4$ were combined and imaged. (F) Similarly, the formulations were prepared separately at $\mathrm{pH} 7.4$ and then combined and imaged. (G) LNP-GNP systems were combined immediately following rapidmixing (in the presence of $25 \%$ ethanol) and the $\mathrm{pH}$ raised to 7.4. (H) LNP-GNP at $\mathrm{pH} 4$ were combined at $\mathrm{pH} 4$ after dialysis to remove solvent, and the $\mathrm{pH}$ was neutralized. Scale bar $=100$ nm. (I) Particle sizes of LNP at pH 4 and $\mathrm{pH} 7.4$ as determined by manual measurement of 200 particles. 2-way ANOVA with Tukey's multiple comparisons correction was used to determine statistical difference. LNP-GNP at $\mathrm{pH} 7.4$ are the same size. For detailed comparisons see Supporting Information. (J) Ten fields of Cryo-TEM images were analysed to determine the number of particles at $\mathrm{pH} 7.4$ that are associated with GNP. The total number of particles counted were 220 per formulation and the percentage of loaded particles was determined. 


\section{CONCLUSIONS}

The results presented in this work show that LNP systems, regardless of payload size, form through fusion of smaller particles as result of the $\mathrm{pH}$ neutralization step rather than the rapidmixing step. This fusion process proceeds until the final particle accumulates sufficient PEG-lipid on the surface to inhibit further fusion. Thus, the LNP size is dictated by the PEG-lipid content, as expected larger proportions of PEG-lipids with smaller PEG moieties are required to achieve a given size. Finally, while the presence of payload does not affect subsequent fusion during $\mathrm{pH}$ neutralization, the size of the payload clearly influences the distribution of payload amongst the LNP formed. In particular, larger payloads such as mRNA, mcDNA and pDNA are more likely to lead to a population of loaded LNP and "empty" LNP.

In summary, the fusion-dependent process of LNP formation leads to a more detailed understanding of the mechanism of formation and the structures of LNP-nucleic acid complexes

formed. Such understanding is basic for the design of more potent LNP formulations of nucleic acid polymers for gene therapy applications. 


\section{CONFLICTS OF INTERST}

The authors have no conflicts to declare.

\section{ACKNOWLEDGEMENTS}

This work was supported by Foundation grant (FDN 148469) from the Canadian Institutes of Health Research, and a British Columbia Innovation Council Ignite grant. DW acknowledges the support by the Swiss National Science Foundation (SNF, Early Postdoc.Mobility Fellowship, Grant No. 174975). RvdM is supported by a VENI Fellowship (\# 14385) from the Netherlands Organization for Scientific Research (NWO). HMGC and BT are funded by the SNF Sinergia grant (\#180257). The authors acknowledge N. Rimann for support with mcDNA production. 


\section{REFERENCES}

1. Press Release, I. Alnylam ${ }^{\circledR}$ Pharmaceuticals, http://investors.alnylam.com/newsreleases/news-release-details/alnylam-announces-first-ever-fda-approval-rnai-therapeutic, Accessed Aug 11, 2018

2. Press Release, I. Alnylam ${ }^{\circledR}$ Pharmaceuticals, http://investors.alnylam.com/newsreleases/news-release-details/alnylam-announces-ema-acceptance-marketingauthorisation, Accessed Feb 1, 2018

3. S. C. Semple, A. Akinc, J. Chen, A. P. Sandhu, B. L. Mui, C. K. Cho, D. W. Sah, D. Stebbing, E. J. Crosley, E. Yaworski, I. M. Hafez, J. R. Dorkin, J. Qin, K. Lam, K. G. Rajeev, K. F. Wong, L. B. Jeffs, L. Nechev, M. L. Eisenhardt, M. Jayaraman, M. Kazem, M. A. Maier, M. Srinivasulu, M. J. Weinstein, Q. Chen, R. Alvarez, S. A. Barros, S. De, S. K. Klimuk, T. Borland, V. Kosovrasti, W. L. Cantley, Y. K. Tam, M. Manoharan, M. A. Ciufolini, M. A. Tracy, A. de Fougerolles, I. MacLachlan, P. R. Cullis, T. D. Madden and M. J. Hope, Nature biotechnology, 2010, 28, 172-176.

4. M. Jayaraman, S. M. Ansell, B. L. Mui, Y. K. Tam, J. Chen, X. Du, D. Butler, L. Eltepu, S. Matsuda, J. K. Narayanannair, K. G. Rajeev, I. M. Hafez, A. Akinc, M. A. Maier, M. A. Tracy, P. R. Cullis, T. D. Madden, M. Manoharan and M. J. Hope, Angewandte Chemie, 2012, 51, 8529-8533.

5. P. R. Cullis and M. J. Hope, Molecular therapy : the journal of the American Society of Gene Therapy, 2017, DOI: 10.1016/j.ymthe.2017.03.013.

6. J. A. Kulkarni, P. R. Cullis and R. van der Meel, Nucleic Acid Ther, 2018, 28, 146-157.

7. L. B. Jeffs, L. R. Palmer, E. G. Ambegia, C. Giesbrecht, S. Ewanick and I. MacLachlan, Pharmaceutical research, 2005, 22, 362-372.

8. N. M. Belliveau, J. Huft, P. J. Lin, S. Chen, A. K. Leung, T. J. Leaver, A. W. Wild, J. B. Lee, R. J. Taylor, Y. K. Tam, C. L. Hansen and P. R. Cullis, Molecular therapy. Nucleic acids, 2012, 1, e37.

9. J. A. Kulkarni, J. L. Myhre, S. Chen, Y. Y. C. Tam, A. Danescu, J. M. Richman and P. R. Cullis, Nanomedicine : nanotechnology, biology, and medicine, 2016, 13, 1377-1387.

10. A. K. Leung, Y. Y. Tam, S. Chen, I. M. Hafez and P. R. Cullis, The journal of physical chemistry. B, 2015, 119, 8698-8706.

11. J. A. Kulkarni, M. M. Darjuan, J. E. Mercer, S. Chen, R. van der Meel, J. L. Thewalt, Y. Y. C. Tam and P. R. Cullis, ACS nano, 2018, 12, 4787-4795.

12. A. K. Leung, I. M. Hafez, S. Baoukina, N. M. Belliveau, I. V. Zhigaltsev, E. Afshinmanesh, D. P. Tieleman, C. L. Hansen, M. J. Hope and P. R. Cullis, J Phys Chem C Nanomater Interfaces, 2012, 116, 18440-18450.

13. M. J. W. Evers, J. A. Kulkarni, R. der Meel, P. R. Cullis, P. Vader and R. M. Schiffelers, Small Methods, 2018, 2, 1700375.

14. A. Akinc, A. Zumbuehl, M. Goldberg, E. S. Leshchiner, V. Busini, N. Hossain, S. A. Bacallado, D. N. Nguyen, J. Fuller, R. Alvarez, A. Borodovsky, T. Borland, R. Constien, A. de Fougerolles, J. R. Dorkin, K. Narayanannair Jayaprakash, M. Jayaraman, M. John, V. Koteliansky, M. Manoharan, L. Nechev, J. Qin, T. Racie, D. Raitcheva, K. G. Rajeev, D. W. Sah, J. Soutschek, I. Toudjarska, H. P. Vornlocher, T. S. Zimmermann, R. Langer and D. G. Anderson, Nature biotechnology, 2008, 26, 561-569.

15. C. Waldner, M. Roose and G. U. Ryffel, BMC Dev Biol, $2009,9,37$. 
16. H. M. Viecelli, R. P. Harbottle, S. P. Wong, A. Schlegel, M. K. Chuah, T. VandenDriessche, C. O. Harding and B. Thony, Hepatology, 2014, 60, 1035-1043.

17. G. Basha, M. Ordobadi, W. R. Scott, A. Cottle, Y. Liu, H. Wang and P. R. Cullis, Molecular therapy. Nucleic acids, 2016, 5, e363.

18. S. Hirota, C. T. de Ilarduya, L. G. Barron and F. C. Szoka, Jr., Biotechniques, 1999, 27, 286-290.

19. J. A. Kulkarni, Y. Y. C. Tam, S. Chen, Y. K. Tam, J. Zaifman, P. R. Cullis and S. Biswas, Nanoscale, 2017, 9, 13600-13609.

20. S. Chen, Y. Y. Tam, P. J. Lin, A. K. Leung, Y. K. Tam and P. R. Cullis, Journal of controlled release : official journal of the Controlled Release Society, 2014, 196, 106-112.

21. S. Chen, Y. Y. Tam, P. J. Lin, M. M. Sung, Y. K. Tam and P. R. Cullis, Journal of controlled release : official journal of the Controlled Release Society, 2016, DOI: 10.1016/j.jconrel.2016.05.059.

22. P. Sengupta, D. Holowka and B. Baird, Biophysical journal, 2007, 92, 3564-3574.

23. R. L. Thurmond, S. W. Dodd and M. F. Brown, Biophysical journal, 1991, 59, 108-113.

24. N. Pardi, S. Tuyishime, H. Muramatsu, K. Kariko, B. L. Mui, Y. K. Tam, T. D. Madden, M. J. Hope and D. Weissman, Journal of controlled release : official journal of the Controlled Release Society, 2015, 217, 345-351.

25. S. J. Eastman, C. Siegel, J. Tousignant, A. E. Smith, S. H. Cheng and R. K. Scheule, Biochimica et biophysica acta, 1997, 1325, 41-62.

26. M. Yanez Arteta, T. Kjellman, S. Bartesaghi, S. Wallin, X. Wu, A. J. Kvist, A. Dabkowska, N. Szekely, A. Radulescu, J. Bergenholtz and L. Lindfors, Proceedings of the National Academy of Sciences of the United States of America, 2018, 115, E3351-E3360.

27. H. M. Grisch-Chan, A. Schlegel, T. Scherer, G. Allegri, R. Heidelberger, P. Tsikrika, M. Schmeer, M. Schleef, C. O. Harding, J. Haberle and B. Thony, Molecular therapy. Nucleic acids, 2017, 7, 339-349. 